\title{
Loop spaces and homotopy operations
}

\author{
by
}

\author{
David B lanc (Haifa)
}

\begin{abstract}
We describe an obstruction theory for an $H$-space $\mathbf{X}$ to be a loop space, in terms of higher homotopy operations taking values in $\pi_{*} \mathbf{X}$. These depend on first algebraically "delooping" the $\Pi$-algebras $\pi_{*} \mathbf{X}$, using the $H$-space structure on $\mathbf{X}$, and then trying to realize the delooped $\Pi$-algebra.
\end{abstract}

1. Introduction. An $H$-space is a topological space $\mathbf{X}$ with a multiplication; the motivating example is a topological group $\mathbf{G}$, which from the point of view of homotopy theory is just a loop space: $\mathbf{G} \simeq \Omega B \mathbf{G}=$ $\mathbf{m a p}_{*}\left(\mathbf{S}^{1}, B \mathbf{G}\right)$. The question of whether a given $H$-space $\mathbf{X}$ is, up to homotopy, a loop space, and thus a topological group (cf. [Mil, §3]), has been studied from a variety of viewpoints - see [A, B, DL, F, H, Ma2, St1, St2, $\mathrm{Ste}, \mathrm{Su}, \mathrm{Z}]$, and the surveys in [St3], [St4, §1], and [Ka, Part II]. Here we address this question from the aspect of homotopy operations, in the classical sense of operations on homotopy groups.

As is well known, the homotopy groups of a space $\mathbf{X}$ have Whitehead products and composition operations defined on them; in addition, there are various higher order operations on $\pi_{*} \mathbf{X}$, such as Toda brackets; and the totality of these actually determine the homotopy type of $\mathbf{X}$ (cf. [Bl3, $\S 7.17]$ ). They should thus enable us - in theory - to determine whether $\mathbf{X}$ is a loop space, up to homotopy. It is the purpose of this note to explain in what sense this can actually be done:

First, we show how an $H$-space structure on $\mathbf{X}$ can be used to define the action of the primary homotopy operations on the shifted homotopy groups $G_{*}=\pi_{*-1} \mathbf{X}$ (which are isomorphic to $\pi_{*} \mathbf{Y}$ if $\mathbf{X} \simeq \Omega \mathbf{Y}$ ). This action will behave properly with respect to composition of operations if $\mathbf{X}$ is homotopyassociative, and will lift to a topological action of the monoid of all maps

1991 Mathematics Subject Classification: Primary 55P45; Secondary 55Q35.

Key words and phrases: loop space, topological group, $H$-space, $\Pi$-algebra, delooping, higher homotopy operations, obstruction theory. 
between spheres if and only if $\mathbf{X}$ is a loop space (see Theorem 5.7 below for the precise statement). The obstructions to having such a topological action may be formulated in the framework of the obstruction theories for realizing $\Pi$-algebras and their morphisms described in [Bl3], which are stated in turn in terms of certain higher homotopy operations:

Theorem A (Theorem 6.24 below). An $H$-group $\mathbf{X}$ is H-equivalent to a loop space if and only if the collection of higher homotopy operations defined in Section 6 below (taking values in homotopy groups) vanish coherently.

The question of whether a given topological space $\mathbf{X}$ supports an $H$-space structure to begin with was addressed in [B14], where a similar obstruction theory, in terms of higher homotopy operations, was defined.

1.1. Notation and conventions. $\mathcal{T}_{*}$ will denote the category of pointed $C W$ complexes with base-point preserving maps, and by a space we shall always mean an object in $\mathcal{T}_{*}$, which will be denoted by a boldface letter: $\mathbf{A}, \mathbf{B}, \ldots, \mathbf{X}, \mathbf{S}^{n}$, and so on. The base-point will be written $* \in \mathbf{X}$. The full subcategory of 0 -connected spaces will be denoted by $\mathcal{T}_{0} . \Delta[n]$ is the standard topological $n$-simplex in $\mathbb{R}^{n+1}$.

The space of Moore loops on $\mathbf{Y} \in \mathcal{T}_{0}$ will be denoted by $\Omega \mathbf{Y}$. This is homotopy equivalent to the usual loop space, that is, the $\operatorname{space}_{\mathbf{m a p}}\left(\mathbf{S}^{1}, \mathbf{Y}\right)$ of pointed maps (see [W, III, Corollary 2.19]). The reduced suspension of $\mathbf{X}$ is denoted by $\Sigma \mathbf{X}$.

$\mathcal{A} b G p$ is the category of abelian groups, and $g r \mathcal{A} b G p$ the category of positively graded abelian groups.

Definition 1.2. $\Delta$ is the category of ordered sequences $\mathbf{n}=\langle 0,1, \ldots, n\rangle$ $(n \in \mathbb{N})$, with order-preserving maps, and $\Delta_{\partial}$ the subcategory having the same objects, but allowing only one-to-one morphisms (so in particular, morphisms from $\mathbf{n}$ to $\mathbf{m}$ exist only for $n \leq m)$. $\Delta^{\mathrm{op}}, \Delta_{\partial}^{\mathrm{op}}$ are the opposite categories.

As usual, a simplicial object over any category $\mathcal{C}$ is a functor $X: \Delta^{\text {op }} \rightarrow$ $\mathcal{C}$; more explicitly, it is a sequence $\left\{X_{n}\right\}_{n=0}^{\infty}$ of objects in $\mathcal{C}$, equipped with face maps $d_{i}: X_{n} \rightarrow X_{n-1}$ and degeneracies $s_{j}: X_{n} \rightarrow X_{n+1}(0 \leq i, j \leq n)$, satisfying the usual simplicial identities ([Ma1, 11.1$])$. We often denote such a simplicial object by $X_{\bullet}$. The category of simplicial objects over $\mathcal{C}$ is denoted by $s \mathcal{C}$.

Similarly, a functor $X: \Delta_{\partial}^{\mathrm{op}} \rightarrow \mathcal{C}$ is called a $\Delta$-simplicial object over $\mathcal{C}$; this is simply a simplicial object without the degeneracies, and will usually be written $X_{\bullet}^{\Delta}$. When $\mathcal{C}=\mathcal{S}$ et, these have been variously called $\Delta$-sets, ss-sets, or restricted simplicial sets in the literature (see [RS]). The category of $\Delta$-simplicial objects over $\mathcal{C}$ is denoted by $\Delta \mathcal{C}$. We shall usually denote the underlying $\Delta$-simplicial object of a simplicial object $Y_{\bullet} \in s \mathcal{C}$ by $Y_{\bullet}^{\Delta} \in \Delta \mathcal{C}$. 
The category of pointed simplicial sets will be denoted by $\mathcal{S}_{*}$ (rather than $s \mathcal{S e t}_{*}$ ); its objects will be denoted by boldface letters $\mathbf{K}, \mathbf{L}, \mathbf{M}, \ldots$ The subcategory of fibrant simplicial sets (Kan complexes) will be denoted by $\mathcal{S}_{*}^{\text {Kan }}$, and that of reduced Kan complexes by $\mathcal{S}_{0}^{\text {Kan }} .|\mathbf{K}| \in \mathcal{T}_{*}$ will denote the geometric realization of a simplicial set $\mathbf{K} \in \mathcal{S}_{*}$, while $S \mathbf{X} \in \mathcal{S}_{*}^{\text {Kan }}$ will denote the singular simplicial set associated with a space $\mathbf{X} \in \mathcal{T}_{*}$. $\mathcal{G}$ is the category of simplicial groups. (See $[\mathrm{Ma} 1, \S \S 3,14,15,17]$ for the definitions.)

For each of the categories $\mathcal{C}=\mathcal{T}_{*}, \mathcal{T}_{0}, \mathcal{S}_{*}^{\text {Kan }}, \mathcal{S}_{0}^{\text {Kan }}$, or $\mathcal{G}$, we will denote by $[\mathbf{X}, \mathbf{Y}]_{\mathcal{C}}$ (or simply $[\mathbf{X}, \mathbf{Y}]$, if there is no danger of confusion) the set of pointed homotopy classes of maps $\mathbf{X} \rightarrow \mathbf{Y}$ (cf. [Ma1, §5] and [K, §3]). The constant pointed map will be written $c_{*}$, or simply $*$. The homotopy category of $\mathcal{C}$, whose objects are those of $\mathcal{C}$, and whose morphisms are homotopy classes of maps in $\mathcal{C}$, will be denoted by $h o \mathcal{C}$. The adjoint functors $S$ and $|\cdot|$ induce equivalences of categories $h o \mathcal{T}_{*} \approx h o \mathcal{S}_{*}^{\text {Kan }}$; similarly $h o \mathcal{S}_{0}^{\text {Kan }} \approx h o \mathcal{G}$ under the adjoint functors $G, \bar{W}$ (see $\S 5.1$ below).

Definition 1.3. An $H$-space structure for a space $\mathbf{X} \in \mathcal{T}_{*}$ is a choice of an $H$-multiplication map $m: \mathbf{X} \times \mathbf{X} \rightarrow \mathbf{X}$ such that $m \circ i=\nabla$, where $i: \mathbf{X} \vee \mathbf{X} \hookrightarrow \mathbf{X} \times \mathbf{X}$ is the inclusion, and $\nabla: \mathbf{X} \vee \mathbf{X} \rightarrow \mathbf{X}$ is the fold map (induced by the identity on each wedge summand). If $\mathbf{X}$ may be equipped with such an $m$, we say that $\langle\mathbf{X}, m\rangle$ (or just $\mathbf{X}$ ) is an $H$-space. (If we only have $m \circ i \sim \nabla$, we can find a homotopic map $m^{\prime} \sim m$ such that $m^{\prime} \circ i=\nabla$, since $\mathbf{X}$ is assumed to be well-pointed.)

An $H$-space $\langle\mathbf{X}, m\rangle$ is homotopy-associative if $m \circ\left(m, \operatorname{id}_{X}\right) \sim m \circ\left(\operatorname{id}_{X}, m\right)$ : $\mathbf{X} \times \mathbf{X} \times \mathbf{X} \rightarrow \mathbf{X}$. It is an $H$-group if it is homotopy-associative and has a (two-sided) homotopy inverse $\iota: \mathbf{X} \rightarrow \mathbf{X}$ with $m \circ\left(\iota \times \operatorname{id}_{X}\right) \circ \Delta \sim c_{*} \sim$ $m \circ\left(\operatorname{id}_{X} \times \iota\right) \circ \Delta$ (where $\Delta: \mathbf{X} \rightarrow \mathbf{X} \times \mathbf{X}$ is the diagonal). In fact, any connected homotopy-associative $H$-space is an $H$-group (cf. [W, X, Theorem 2.2]).

If $\langle\mathbf{X}, m\rangle$ and $\langle\mathbf{Y}, n\rangle$ are two $H$-spaces, a map $f: \mathbf{X} \rightarrow \mathbf{Y}$ is called an $H$-map if $n \circ(f \times f) \sim f \circ m: \mathbf{X} \times \mathbf{X} \rightarrow \mathbf{Y}$. The set of pointed homotopy classes of $H$-maps $\mathbf{X} \rightarrow \mathbf{Y}$ will be denoted by $[\mathbf{X}, \mathbf{Y}]_{H}$.

One similarly defines $H$-simplicial sets and simplicial $H$-maps in the category $\mathcal{S}_{*}$.

1.4. Organization. In Section 2 we review some background material on $\Pi$-algebras, and in Section 3 we explain how an $H$-space structure on $\mathbf{X}$ determines the $\Pi$-algebra structure of its potential delooping. In Section 4 we provide further background on $(\Delta$-)simplicial spaces and $\Pi$-algebras, and bisimplicial groups. In Section 5 we show, in the context of simplicial groups, that the $\Pi$-algebra structure on $\pi_{*-1} \mathbf{X}$ can be made "topological" if and only if $\mathbf{X}$ is a loop space (Theorem 5.7). 
Finally, in Section 6 we recall the obstruction theory of [Bl3] for realizing $\Pi$-algebras in terms of rectifying $(\Delta$-)simplicial spaces, and explain how it applies to the recognition of loop spaces (see Theorem 6.24). We also simplify the general obstruction theory in question, by showing that it suffices to rectify the underlying $\Delta$-simplicial space associated with a free simplicial $\Pi$-algebra resolution (Proposition 6.14).

I would like to thank the referee for his comments.

2. $\Pi$-algebras. In this section we briefly recall some facts on the primary homotopy operations.

Definition 2.1. A $\Pi$-algebra is a graded group $G_{*}=\left\{G_{k}\right\}_{k=1}^{\infty}$ (abelian in degrees $>1$ ), together with an action on $G_{*}$ of the primary homotopy operations (i.e., compositions and Whitehead products, including the " $\pi_{1}$ action" of $G_{1}$ on the higher $G_{n}$ 's, as in [W, X, $\left.\S 7\right]$ ), satisfying the usual universal identities. See $[\mathrm{Bl} 1, \S 3]$ or $[\mathrm{Bl} 2, \S 2.1]$ for a more explicit description. The category of $\Pi$-algebras (with the obvious morphisms) will be denoted by $\Pi$-Alg.

Definition 2.2. We say that a space $\mathbf{X}$ realizes a $\Pi$-algebra $G_{*}$ if there is an isomorphism of $\Pi$-algebras $G_{*} \cong \pi_{*} \mathbf{X}$. (There may be non-homotopy equivalent spaces realizing the same $\Pi$-algebra - cf. [Bl3, §7.18].) Similarly, a morphism of $\Pi$-algebras $\phi: \pi_{*} \mathbf{X} \rightarrow \pi_{*} \mathbf{Y}$ (between realizable $\Pi$-algebras) is realizable if there is a map $f: \mathbf{X} \rightarrow \mathbf{Y}$ such that $\pi_{*} f=\phi$.

Definition 2.3. The free $\Pi$-algebras are those isomorphic to $\pi_{*} \mathbf{W}$, for some (possibly infinite) wedge of spheres $\mathbf{W}$; we say that $\pi_{*} \mathbf{W}$ is generated by a graded set $L_{*}=\left\{L_{k}\right\}_{k=1}^{\infty}$, and write $\pi_{*} \mathbf{W} \cong F\left(L_{*}\right)$, if $\mathbf{W}=$ $\bigvee_{k=1}^{\infty} \bigvee_{x \in L_{k}} \mathbf{S}_{x}^{k}$

FACT 2.4. If we let $\Pi$ denote the homotopy category of wedges of spheres, and $\mathcal{F} \subset \Pi$-Alg the full subcategory of free $\Pi$-algebras, then $\pi_{*}: \Pi \rightarrow \mathcal{F}$ is an equivalence of categories. Note that any $\Pi$-algebra morphism $\phi: G_{*} \rightarrow$ $G_{*}^{\prime}$ is uniquely realizable if $G_{*}$ is a free $\Pi$-algebra.

For future reference we note the following:

Lemma 2.5. If $A_{*}, B_{*} \in \mathcal{F}$ are free $\Pi$-algebras and $A_{*} \stackrel{i}{\hookrightarrow} B_{*} \stackrel{r}{\rightarrow} A_{*}$ is a retraction $\left(r \circ i=\mathrm{id}_{A_{*}}\right)$, then there is a free $\Pi$-algebra $C_{*} \in \mathcal{F}$ such that $B_{*}=A_{*} \amalg C_{*}$.

Proof. Let $Q: \Pi$-Alg $\rightarrow g r \mathcal{A} b G p$ be the "indecomposables" functor (so $Q\left(\pi_{*} \mathbf{W}\right) \cong H_{*}(W ; \mathbb{Z})$ - see $\left.[\mathrm{Bl} 1, \S 2.2 .1]\right)$; then $Q\left(A_{*}\right)$ and $Q\left(B_{*}\right)$ are free abelian groups, and as $Q\left(A_{*}\right) \stackrel{Q(i)}{\hookrightarrow} Q\left(B_{*}\right) \stackrel{Q(r)}{\longrightarrow} Q\left(A_{*}\right)$ is a retraction in $\mathcal{A} b G p$, there is a graded free abelian group $E_{*}$ such that $Q\left(B_{*}\right)=Q\left(A_{*}\right) \oplus E_{*}$. 
Choosing graded sets $\left\{e_{\gamma}\right\}_{\gamma \in \Gamma}$ of generators for $E_{*}$ (in degree 1, choose generators for the free group $\left.\operatorname{Ker}(r) \subseteq B_{*}\right)$, and setting $C_{*}=F\left(\left\{e_{\gamma}\right\}_{\gamma \in \Gamma}\right)$, yields the required decomposition (by the Hurewicz Theorem).

Definition 2.6. Let $T: \Pi$-Alg $\rightarrow \Pi$-Alg be the "free $\Pi$-algebra" comonad (cf. [M, VI, §1]), defined by $T G_{*}=\coprod_{k=1}^{\infty} \coprod_{g \in G_{k} \backslash\{0\}} \pi_{*} \mathbf{S}_{(g)}^{k}$. The counit $\varepsilon=\varepsilon_{G_{*}}: T G_{*} \rightarrow G_{*}$ is defined by $\iota_{(g)}^{k} \mapsto g$ (where $\iota_{(g)}^{k}$ is the canonical generator of $\left.\pi_{*} \mathbf{S}_{(g)}^{k}\right)$, and the comultiplication $\vartheta=\vartheta_{G_{*}}: T G_{*} \hookrightarrow T^{2} G_{*}$ is induced by the natural transformation $\bar{\vartheta}:\left.\operatorname{id}_{\mathcal{F}} \rightarrow T\right|_{\mathcal{F}}$ defined by $x_{k} \mapsto \iota_{\left(x_{k}\right)}^{k}$.

Definition 2.7. An abelian $\Pi$-algebra is one for which all Whitehead products vanish.

These are indeed the abelian objects of $\Pi-A l g$ - see [Bl2, §2]. If $\mathbf{X}$ is an $H$-space, then $\pi_{*} \mathbf{X}$ is an abelian $\Pi$-algebra (cf. [W, X, (7.8)]).

3. Secondary $\Pi$-algebra structure. We now describe how an $H$ space structure on $\mathbf{X}$ determines the $\Pi$-algebra structure of a (potential) classifying space.

3.1. The James construction. For any $\mathbf{X} \in \mathcal{T}_{*}$, let $J \mathbf{X}$ be the James reduced product construction, with $\lambda: J \mathbf{X} \rightarrow \Omega \Sigma \mathbf{X}$ the homotopy equivalence of [W, VII, (2.6)], and $j_{X}: \mathbf{X} \hookrightarrow J \mathbf{X}$ and $i_{X}: \mathbf{X} \hookrightarrow \Omega \Sigma \mathbf{X}$ the natural inclusions.

If $\langle\mathbf{X}, m\rangle$ is an $H$-space, then there is a retraction $\bar{m}: J \mathbf{X} \rightarrow \mathbf{X}$ (with $\left.\bar{m} \circ j_{X}=\operatorname{id}_{X}\right)$, defined by

$$
\bar{m}\left(x_{1}, x_{2}, \ldots, x_{n}\right)=m\left(\ldots m\left(m\left(x_{1}, x_{2}\right), x_{3}\right), \ldots, x_{n}\right)
$$

(cf. [J, Theorem 1.8]).

Definition 3.3. Let $\mathbf{X}$ be an $H$-space. Given homotopy classes $\alpha \in$ $[\Sigma \mathbf{A}, \Sigma \mathbf{B}]$ and $\beta \in[\mathbf{B}, \mathbf{X}]$, we define the derived composition $\alpha \star \beta \in[\mathbf{A}, \mathbf{X}]$ as follows:

Choose representatives $f: \Sigma \mathbf{A} \rightarrow \Sigma \mathbf{B}$ and $g: \mathbf{B} \rightarrow \mathbf{X}$ for $\alpha, \beta$ respectively, and let $\lambda^{-1}: \Omega \Sigma \mathbf{X} \rightarrow J \mathbf{X}$ be any homotopy inverse to $\lambda$. Then $\alpha \star \beta$ is represented by the composite

$$
\mathbf{A} \stackrel{i_{A}}{\longrightarrow} \Omega \Sigma \mathbf{A} \stackrel{\Omega \alpha}{\longrightarrow} \Omega \Sigma \mathbf{B} \stackrel{\lambda^{-1}}{\longrightarrow} J \mathbf{B} \stackrel{J \beta}{\longrightarrow} J \mathbf{X} \stackrel{\bar{m}}{\longrightarrow} \mathbf{X} .
$$

FACT 3.4. Note that if $\alpha=\Sigma \bar{\alpha}$ for some $\bar{\alpha}: \mathbf{A} \rightarrow \mathbf{B}$, then $\alpha \star \beta=\bar{\alpha}^{\#} \beta$ (this is well-defined, because $\mathbf{X}$ is an $H$-space).

We shall be interested in the case where $\mathbf{B}$ is a wedge of spheres and $\mathbf{A}=\mathbf{S}^{n}$, so $\star$ assigns a class $\omega \star\left(\beta_{1}, \ldots, \beta_{k}\right) \in \pi_{n} \mathbf{X}$ to any $k$-ary homotopy operation $\omega^{\#}: \pi_{n_{1}+1}(-) \times \ldots \times \pi_{n_{k}+1}(-) \rightarrow \pi_{n+1}(-)$ and collection of elements $\beta_{i} \in \pi_{n_{i}} \mathbf{X}(i=1, \ldots, k)$. 
In particular, if $\omega: \mathbf{S}^{p+q+1} \rightarrow \mathbf{S}^{p+1} \vee \mathbf{S}^{q+1}$ represents the Whitehead product, one may define a "Samelson product" $\omega \star(-,-): \pi_{p} \mathbf{X} \times \pi_{q} \mathbf{X} \rightarrow$ $\pi_{p+q} \mathbf{X}$ for any $H$-space $\mathbf{X}$, even without assuming associativity or the existence of a homotopy inverse (compare [W, X, §5]).

However, in general this $\omega \star(-,-)$ need not enjoy any of the usual properties of the Samelson product (bi-additivity, graded-commutativity, Jacobi identity - cf. [W, X, Theorems $5.1 \&$ 5.4]). To ensure that they hold, one needs further assumptions on $\mathbf{X}$.

First, we note the following homotopy version of [W, VII, Theorem 2.5], which appears to be folklore:

Lemma 3.5. If $\langle\mathbf{X}, m\rangle$ is a homotopy-associative $H$-space, then any map $f: \mathbf{A} \rightarrow \mathbf{X}$ extends to an $H$-map $\widehat{f}: J \mathbf{A} \rightarrow \mathbf{X}$, which is unique up to homotopy.

$$
\begin{aligned}
& \text { Proof. Given } f: \mathbf{A} \rightarrow \mathbf{X} \text {, define } \widehat{f}: J \mathbf{A} \rightarrow \mathbf{X} \text { by } \\
& \qquad \widehat{f}\left(x_{1}, \ldots, x_{r}\right)=m\left(\ldots m\left(m\left(f\left(x_{1}\right), f\left(x_{2}\right)\right), f\left(x_{3}\right)\right), \ldots, f\left(x_{r}\right)\right) .
\end{aligned}
$$

This is an $H$-map by [N, Lemma 1.4]. Now let $\widehat{g}: J \mathbf{A} \rightarrow \mathbf{X}$ be another $H$-map, with a homotopy $H: f \simeq g:=\widehat{g} \circ j_{A}$. Since $\widehat{g}$ is an $H$-map, there is a homotopy $G: n \circ(\widehat{g} \times \widehat{g}) \simeq \widehat{g} \circ m$ (where $m: J \mathbf{A} \times J \mathbf{A} \rightarrow J \mathbf{A}$ is the $H$-multiplication). Moreover, by [N, Lemma 1.3(a)] we may assume $G$ is stationary on $J \mathbf{A} \vee J \mathbf{A}$.

For each $r \geq 0$, let $J_{r} \mathbf{A}$ denote the $r$ th stage in the construction of $J \mathbf{A}$, with $j_{r}^{s}: J_{s} \mathbf{A} \hookrightarrow J_{r} \mathbf{A}$ and $j^{s}: J_{s} \mathbf{A} \hookrightarrow J \mathbf{A}$ the inclusions, starting with $J_{0} \mathbf{A}=*$ and $J_{1} \mathbf{A}=\mathbf{A}$. We define $T_{r} \mathbf{A}$ to be the pushout in the following diagram:

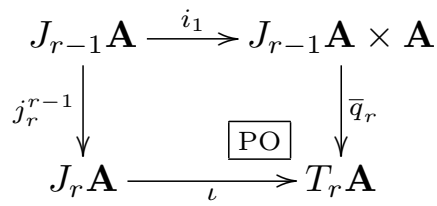

for $r \geq 1$ (so $T_{1} \mathbf{A}=\mathbf{A} \vee \mathbf{A}$ ); then $J_{r+1} \mathbf{A}$ is the pushout in

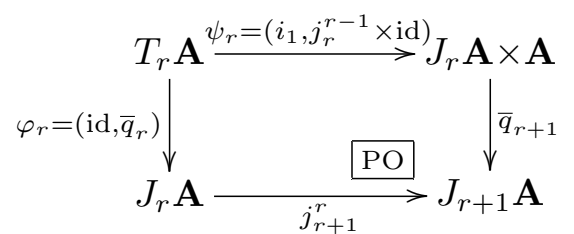

Now let $\widehat{f}_{r}=\left.\widehat{f}\right|_{J_{r} \mathbf{A}}$ and $\widehat{g}_{r}=\left.\widehat{g}\right|_{J_{r} \mathbf{A}}$; we shall extend $H: f \simeq g$ to a homotopy $\widehat{H}: \widehat{f} \simeq \widehat{g}$ by inductively constructing homotopies $\widehat{H}_{r}: \widehat{f}_{r} \simeq \widehat{g}_{r}$ (starting with $\widehat{H}_{1}=H$ ) such that $\left.\widehat{H}_{r}\right|_{J_{r-1}} \mathbf{A}=\widehat{H}_{r-1}$ : let $n_{r}: \mathbf{X}^{r} \rightarrow \mathbf{X}$ 
denote the $n$-fold multiplication $\left.n_{r}\left(x_{1}, \ldots, x_{r}\right)=n\left(\ldots n\left(x_{1}, x_{2}\right), \ldots\right), x_{r}\right)$ and $q_{r}: \mathbf{A}^{r} \rightarrow J_{r} \mathbf{A}$ the quotient map, so that $n^{r} \circ f^{r}=\widehat{f}_{r} \circ q_{r}$.

As a first approximation, define $\bar{H}_{r+1}: \widehat{f}_{r} \times f \simeq \widehat{g}_{r} \times g$ on $J_{r} \mathbf{A} \times \mathbf{A}$ in the above pushout to be the sum of homotopies $\bar{H}_{r+1}=n \circ\left(\widehat{H}_{r} \times H\right)+G \circ$ $\left(j^{r} \times j_{A}\right)$. This does not quite agree with $\widehat{H}_{r} \circ \varphi_{r}$ on $T_{r} \mathbf{A}$, but since $G$ is stationary on $J \mathbf{A} \vee J \mathbf{A}$ we have $\left.\bar{H}_{r+1}\right|_{J_{r} \mathbf{A}}=n \circ\left(\widehat{H}_{r} \times\right.$ id $)+($ stationary $)=$ $\widehat{H}_{r}+$ (stationary) and $\left.\bar{H}_{r+1}\right|_{J_{r-1}} \mathbf{A} \times \mathbf{A}=n \circ\left(\widehat{H}_{r-1} \times H\right)+G \circ\left(j^{r-1} \times j_{A}\right)=\bar{H}_{r}$.

As $\widehat{H}_{1}=H$, we see that $\left.\bar{H}_{2}\right|_{T_{2} \mathbf{A}}=(H+$ (stationary), $H)$, while $H \circ \varphi_{1}$ $=(H, H)$. Thus we may assume by induction that there is a homotopy of homotopies $F:\left.\bar{H}_{r+1}\right|_{T_{r} \mathbf{A}} \simeq \widehat{H}_{r} \circ \varphi_{r}$. Since $T_{r} \mathbf{A} \hookrightarrow J_{r} \mathbf{A} \times \mathbf{A}$ is a cofibration, the inclusion

$$
T_{r} \mathbf{A} \times I^{2} \cup\left(J_{r} \mathbf{A} \times \mathbf{A}\right) \times(\{0,1\} \times I \cup I \times\{0\}) \hookrightarrow\left(J_{r} \mathbf{A} \times \mathbf{A}\right) \times I^{2}
$$

is a trivial cofibration, and thus we may use the homotopy extension property to obtain a new homotopy $\widetilde{F}$ on $\left(J_{r} \mathbf{A} \times \mathbf{A}\right) \times I^{2}$ which restricts to $\widetilde{H}_{r+1}: \widehat{f}_{r} \times f \simeq \widehat{g}_{r} \times g$ on $J_{r} \mathbf{A} \times \mathbf{A} \times I \times\{1\}$, such that $\widetilde{H}_{r+1}$ extends $\widehat{H}_{r} \circ \varphi_{r}$, and thus may be combined with $\widehat{H}_{r}$ to define a homotopy $\widehat{H}_{r+1}$ as required.

Corollary 3.6. If $\mathbf{X}$ is a homotopy-associative $H$-space, then for any $\mathbf{A} \in \mathcal{T}_{*}$ the inclusion $j_{A}: \mathbf{A} \rightarrow J \mathbf{A}$ induces a bijection $j_{A}^{*}:[J \mathbf{A}, X]_{H} \cong$ $[\mathbf{A}, \mathbf{X}]_{\mathcal{T}_{*}}$.

Proof. Since $\mathbf{X}$ is a homotopy-associative $H$-space, the retraction $\bar{n}=$ $\widehat{\mathrm{id}}_{X}: J \mathbf{X} \rightarrow \mathbf{X}$ is an $H$-map, by Lemma 3.5 , so we may define $\phi:[\mathbf{A}, \mathbf{X}]_{\mathcal{T}_{*}} \rightarrow$ $[J \mathbf{A}, \mathbf{X}]_{H}$ by $\phi([f])=[\bar{m} \circ J(f)]$, and clearly $j_{A}^{*}(\phi([f]))=[\bar{m} \circ J(f)$ $\left.\circ j_{A}\right]=[f]$. On the other hand, given an $H$-map $g: J \mathbf{A} \rightarrow \mathbf{X}$ we have $\bar{m} \circ J\left(g \circ j_{\mathbf{A}}\right) \circ j_{A} \simeq g \circ j_{A}$, which implies that $\bar{m} \circ J\left(g \circ j_{\mathbf{A}}\right) \simeq g$ by Lemma 3.5 again. Thus also $\phi\left(j_{A}^{*}([g])\right)=[g]$.

3.7. Notation. If $\mathbf{X}$ is a homotopy-associative $H$-space, we shall write $\pi_{t}^{H} \mathbf{X}$ for $\left[\Omega \mathbf{S}^{t}, \mathbf{X}\right]_{H}=\left[J \mathbf{S}^{t-1}, \mathbf{X}\right]_{H} \cong \pi_{t-1} \mathbf{X}$.

Proposition 3.8. If $\mathbf{X}$ is a homotopy-associative $H$-space, then

$$
\alpha \star(\beta \star \gamma)=\left(\alpha^{\#} \beta\right) \star \gamma
$$

for any $\alpha \in[\Sigma \mathbf{A}, \Sigma \mathbf{B}], \beta \in[\Sigma \mathbf{B}, \Sigma \mathbf{C}]$, and $\gamma \in[\mathbf{C}, \mathbf{X}]$.

Proof. It suffices to consider $\alpha=\mathrm{id}_{\Sigma B}$, and so to show that

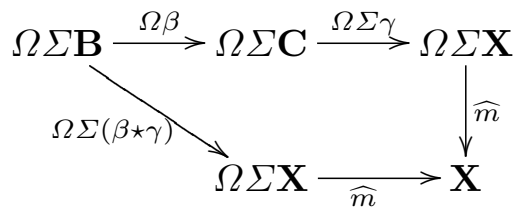


commutes up to homotopy (where $\widehat{m}$ is the composite $\Omega \Sigma \mathbf{X} \stackrel{\lambda^{-1}}{\longrightarrow} J \mathbf{X}$ $\stackrel{\bar{m}}{\longrightarrow} \mathbf{X})$ - or, since $\beta \star \gamma$ is defined to be the composite $\widehat{m} \circ \Omega \Sigma \gamma \circ \Omega \beta \circ i_{B}$, that the two composites $\phi=\widehat{m} \circ \Omega \Sigma \gamma \circ \Omega \beta$ and $\psi=\widehat{m} \circ \Omega \Sigma \widehat{m} \circ(\Omega \Sigma)^{2} \gamma \circ$ $\Omega \Sigma \Omega \beta \circ \Omega \Sigma i_{B}$ are homotopic.

Now if $\mathbf{X}$ is a homotopy-associative $H$-space, then $\widehat{m}$ is an $H$-map by Lemma 3.5, so $\phi, \psi: \Omega \Sigma \mathbf{B} \rightarrow \mathbf{X}$ are $H$-maps. By Corollary 3.6 it suffices to check that $\phi \circ i_{B} \sim \psi \circ i_{B}$-i.e., that $\widehat{m} \circ \Omega \Sigma \gamma \circ \Omega \beta \circ i_{B}$ is homotopic to the composition of

$$
\begin{aligned}
& \mathbf{B} \stackrel{i_{B}}{\longrightarrow} \Omega \Sigma \mathbf{B} \stackrel{\Omega \Sigma i_{B}}{\longrightarrow}(\Omega \Sigma)^{2} \mathbf{B} \stackrel{\Omega \Sigma \Omega \beta}{\longrightarrow}(\Omega \Sigma)^{2} \mathbf{C} \\
& \stackrel{(\Omega \Sigma)^{2} \gamma}{\longrightarrow}(\Omega \Sigma)^{2} \mathbf{X} \stackrel{\Omega \Sigma \widehat{m}}{\longrightarrow} \Omega \Sigma \mathbf{X ~} \stackrel{\widehat{m}}{\longrightarrow} \mathbf{X} .
\end{aligned}
$$

But $\Omega \Sigma \gamma \circ \Omega \beta \circ i_{B}$ is adjoint to $(\Sigma \gamma) \circ \beta$, while the composition of $\mathbf{B} \stackrel{i_{B}}{\longrightarrow} \Omega \Sigma \mathbf{B} \stackrel{\Omega \Sigma i_{B}}{\longrightarrow}(\Omega \Sigma)^{2} \mathbf{B} \stackrel{\Omega \Sigma \Omega \beta}{\longrightarrow}(\Omega \Sigma)^{2} \mathbf{C} \stackrel{(\Omega \Sigma)^{2} \gamma}{\longrightarrow}(\Omega \Sigma)^{2} \mathbf{X} \stackrel{\Omega \Sigma \widehat{m}}{\longrightarrow} \Omega \Sigma \mathbf{X}$ is adjoint to $\Sigma\left(\widehat{m} \circ \Omega \Sigma \gamma \circ \Omega \beta \circ i_{B}\right)$ which is equal to $\Sigma(\widehat{m} \circ(\widetilde{\delta}) \circ \beta)$ (where $\tilde{f}$ denotes the adjoint of $f$ ). Since for any $f: \mathbf{Y} \rightarrow \mathbf{Z}$ the adjoint of $\Sigma f$ is $\Omega \Sigma f \circ i_{Y}$, we see $\widehat{m} \circ \widetilde{\Sigma f} \sim f$, which completes the proof.

It is readily verified that when $\mathbf{X} \simeq \Omega \mathbf{Y}$, the secondary composition is the adjoint of the usual composition in $\pi_{*} \mathbf{Y}$; thus we have:

Corollary 3.9. If $\mathbf{X}$ is an $H$-group, then the graded abelian group $G_{*}$ defined by $G_{k}:=\pi_{k}^{H} \mathbf{X} \cong \pi_{k-1} \mathbf{X}$ (with $\bar{\gamma} \in G_{k}$ corresponding to $\gamma \in \pi_{k-1} \mathbf{X}$ ) has a II-algebra structure defined by the derived compositions; that is, if $\psi \in \pi_{k}\left(\mathbf{S}^{t_{1}} \vee \ldots \vee \mathbf{S}^{t_{n}}\right)$ and $\bar{\gamma}_{j} \in G_{t_{j}}$ for $1 \leq j \leq n$, then

$$
\psi^{\#}\left(\bar{\gamma}_{1}, \ldots, \bar{\gamma}_{n}\right):=\overline{\psi \star\left(\gamma_{1}, \ldots, \gamma_{n}\right)} \in G_{k} .
$$

If $\mathbf{X} \simeq \Omega \mathbf{Y}$, then $G_{*}$ is isomorphic to $\pi_{*} \mathbf{Y}$ as a $\Pi$-algebra.

Definition 3.10. For any $H$-group $\langle\mathbf{X}, m\rangle$, the $\Pi$-algebra structure on the graded abelian group $G_{*}$ of Corollary 3.9 will be called the delooping of $\pi_{*} \mathbf{X}$, and denoted by $\Omega^{-1} \pi_{*} \mathbf{X}$ (so in particular $\Omega^{-1} \pi_{*} \Omega \mathbf{Y} \cong \pi_{*} \mathbf{Y}$ ).

Remark 3.11. Note that Corollary 3.9 provides us with an algebraic obstruction to delooping a space $\mathbf{X}$ : if there is no way of putting a $\Pi$-algebra structure on the graded abelian group $G_{*}=\pi_{*-1} \mathbf{X}$ which is consistent with Fact 3.4, then $\mathbf{X}$ is not a loop space, or even a homotopy-associative $H$-space. (This is of course assuming that the $\Pi$-algebra $\pi_{*} \mathbf{X}$ is abelian-otherwise X cannot even be an $H$-space.)

Example 3.12 . Consider the $\Pi$-algebra $G_{*}$ defined by $G_{2}=\mathbb{Z}\langle x\rangle$ (i.e., $x$ generates the cyclic group $\left.G_{2}\right), G_{3}=\mathbb{Z} / 2\left\langle\eta_{2}^{\#} x\right\rangle, G_{4}=\mathbb{Z} / 2\left\langle\eta_{3}^{\#} \eta_{2}^{\#} x\right\rangle$, and $G_{5}=\mathbb{Z} / 2\left\langle\eta_{4}^{\#} \eta_{3}^{\#} \eta_{2}^{\#} x\right\rangle$, with $G_{t}=0$ for $t \neq 2,3,4,5$ and all Whitehead products zero. 
There can be no homotopy-associative $H$-space $\mathbf{X}$ with $\pi_{*} \mathbf{X} \cong G_{*}$, since the $\Pi$-algebra $G_{*}^{\prime}=\Omega^{-1} G_{*}$ cannot be defined consistently: we would have $G_{3}^{\prime}=\mathbb{Z}\langle\bar{x}\rangle, G_{4}^{\prime}=\mathbb{Z} / 2\left\langle\eta_{3}^{\#} \bar{x}\right\rangle, G_{5}^{\prime}=\mathbb{Z} / 2\left\langle\eta_{4}^{\#} \eta_{3}^{\#} \bar{x}\right\rangle$, and $G_{6}^{\prime}=\mathbb{Z} / 2\left\langle\eta_{5}^{\#} \eta_{4}^{\#} \eta_{3}^{\#} \bar{x}\right\rangle$ by Fact 3.4 ; but $\pi_{6} \mathbf{S}^{3}=\mathbb{Z} / 12\langle\alpha\rangle$ with $6 \alpha=\eta_{5}^{\#} \eta_{4}^{\#} \eta_{3}$, and thus $\alpha^{\#} \bar{x} \in G_{6}^{\prime}$ cannot be defined consistently with the fact that $(6 \alpha)^{\#} \bar{x} \neq 0$.

(We do not claim that $G_{*}$ is realizable; but the obstructions to realizing $G_{*}$ by a space $\mathbf{X} \in \mathcal{T}_{*}$ require secondary (or higher order) information, while the obstructions to its realization by an $H$-group are primary.)

4. Simplicial spaces and $\Pi$-algebras. We next recall some background on $(\Delta$-)simplicial spaces and $\Pi$-algebras, and bisimplicial groups:

DEFINITION 4.1. Recall that a simplicial object over a category $\mathcal{C}$ is a functor $X: \Delta^{\mathrm{op}} \rightarrow \mathcal{C}(\S 1.2)$; an augmented simplicial object $X_{\bullet} \rightarrow A$ over $\mathcal{C}$ is a simplicial object $X_{\bullet} \in s \mathcal{C}$, together with an augmentation $\varepsilon: X_{0} \rightarrow A$ in $\mathcal{C}$ such that

$$
\varepsilon \circ d_{1}=\varepsilon \circ d_{0}
$$

Similarly for an augmented $\Delta$-simplicial object.

Definition 4.3. A simplicial $\Pi$-algebra $A_{*}$ is called free if for each $n \geq 0$ there is a graded set $T^{n} \subseteq\left(A_{*}\right)_{n}$ such that $\left(A_{*}\right)_{n}$ is the free $\Pi$-algebra generated by $T^{n}$, and each degeneracy map $s_{j}:\left(A_{*}\right)_{n} \rightarrow\left(A_{*}\right)_{n+1}$ takes $T^{n}$ to $T^{n+1}$.

A free simplicial resolution of a $\Pi$-algebra $G_{*}$ is defined to be an augmented simplicial $\Pi$-algebra $A_{*} \bullet \rightarrow G_{*}$ such that $A_{* \bullet}$ is a free simplicial $\Pi$-algebra, the homotopy groups of the simplicial group $A_{k} \bullet$ vanish in dimensions $n \geq 1$, and the augmentation induces an isomorphism $\pi_{0} A_{k} \cong G_{k}$.

Such resolutions always exist, for any $\Pi$-algebra $G_{*}$ - see [Q1, II, §4], or the explicit construction in [Bl1, §4.3].

4.4. Realization. Let $\mathbf{W}_{\bullet} \in s \mathcal{T}_{*}$ be a simplicial space; its realization (or homotopy colimit) is a space $\left|\mathbf{W}_{\bullet}\right| \in \mathcal{T}_{*}$ constructed by making identifications in $\coprod_{n=0}^{\infty} \mathbf{W}_{n} \times \Delta[n]$ according to the face and degeneracy maps of W. (cf. [S1, §1]). There is also a modified realization $\left\|\mathbf{W}_{\bullet}\right\| \in \mathcal{T}_{*}$, defined similarly, but without making the identifications along the degeneracies; for "good" simplicial spaces (which include all those we shall consider here) one has $\left\|\mathbf{W}_{\bullet}\right\| \stackrel{\simeq}{\rightarrow}\left|\mathbf{W}_{\bullet}\right|$ (cf. [S2, App. A]). Of course, $\left\|\mathbf{W}_{\bullet}^{\Delta}\right\|$ is also defined for $\Delta$-simplicial spaces $\mathbf{W}_{\bullet}^{\Delta} \in \Delta \mathcal{T}_{*}$.

For any reasonable simplicial space $\mathbf{W}$., there is a first quadrant spectral sequence with

$$
E_{s, t}^{2}=\pi_{s}\left(\pi_{t} \mathbf{W}_{\bullet}\right) \Rightarrow \pi_{s+t}\left|\mathbf{W}_{\bullet}\right|
$$

(see [BF, Thm. B.5] and [BL, App.]). 
Definition 4.6. For any connected $\mathbf{X} \in \mathcal{T}_{*}$, an augmented simplicial space $\mathbf{W}_{\bullet} \rightarrow \mathbf{X}$ is called a resolution of $\mathbf{X}$ by spheres if each $\mathbf{W}_{n}$ is homotopy equivalent to a wedge of spheres, and $\pi_{*} \mathbf{W} \bullet \rightarrow \pi_{*} \mathbf{X}$ is a free simplicial resolution of $\Pi$-algebras (Def. 4.3).

Using the above spectral sequence, we see that the natural map $\mathbf{W}_{0} \rightarrow$ $\left|\mathbf{W}_{\bullet}\right|$ then induces an isomorphism $\pi_{*} \mathbf{X} \cong \pi_{*}\left|\mathbf{W}_{\bullet}\right|$, so $\left|\mathbf{W}_{\bullet}\right| \simeq \mathbf{X}$.

5. A simplicial group version. For our purposes it will be convenient to work at times in the category $\mathcal{G}$ of simplicial groups. First, we recall some basic definitions and facts:

5.1. Simplicial groups. Let $F: \mathcal{S}_{*} \rightarrow \mathcal{G}$ denote the free group functor of $[\mathrm{Mi2}, \S 2]$; this is the simplicial version of the James construction, and in particular $|F \mathbf{K}| \simeq J|\mathbf{K}|$.

Let $G: \mathcal{S}_{*} \rightarrow \mathcal{G}$ be Kan's simplicial loop functor (cf. [Ma1, Def. 26.3]), with $\bar{W}: \mathcal{G} \rightarrow \mathcal{S}_{0}^{\text {Kan }}$ its adjoint, the Eilenberg-Mac Lane classifying space functor (cf. [Ma1, §21]).

Then $|G \mathbf{K}| \simeq \Omega|\mathbf{K}|$ and $|\mathbf{K}| \simeq|\bar{W} G \mathbf{K}|$. Moreover, unlike $\mathcal{T}_{*}$, where we have only a (weak) homotopy equivalence, in $\mathcal{G}$ there is a canonical isomorphism $\phi: F \mathbf{K} \cong G \Sigma \mathbf{K}$ (cf. [C, Prop. 4.15]), and there are natural bijections

$$
\begin{aligned}
\operatorname{Hom}_{\mathcal{S}_{*}}(\Sigma \mathbf{L}, \bar{W} F \mathbf{K}) \cong & \operatorname{Hom}_{\mathcal{G}}(G \Sigma \mathbf{L}, F \mathbf{K}) \\
& \stackrel{\phi^{*}}{\longrightarrow} \operatorname{Hom}_{\mathcal{G}}(F \mathbf{L}, F \mathbf{K}) \cong \operatorname{Hom}_{\mathcal{S}_{*}}(\mathbf{L}, F \mathbf{K})
\end{aligned}
$$

for any $\mathbf{L} \in \mathcal{S}_{*}$ (induced by the adjunctions), and similarly for homotopy classes of maps.

Thus, we may think of $F \mathbf{S}^{n}$ as the simplicial group analogue of the $n$-sphere; in particular, if $\mathbf{K}$ is in $\mathcal{G}$, or even if $\mathbf{K}$ is just an associative $H$ simplicial set which is a Kan complex, we shall write $\pi_{t}^{H} \mathbf{K}$ for $\left[F \mathbf{S}^{t-1}, \mathbf{K}\right]_{H}$ (compare §3.7). Similarly, $F \mathbf{e}^{n}$ is the $\mathcal{G}$ analogue of the $n$-disc in the sense that any nullhomotopic map $f: F \mathbf{S}^{n-1} \rightarrow \mathbf{K}$ extends to $F \mathbf{e}^{n}$.

R e mark 5.3. The same facts as in $\S 4.4$ hold also if we consider bisimplicial groups (which we shall think of as simplicial objects $\mathbf{G}_{\bullet} \in s \mathcal{G}$ ) instead of simplicial spaces. In this case the realization $\left|\mathbf{W}_{\bullet}\right|$ should be replaced by the diagonal $\operatorname{diag}\left(\mathbf{G}_{\bullet}\right)$, and the spectral sequence corresponding to (4.5), with

$$
E_{s, t}^{2}=\pi_{s}\left(\pi_{t} \mathbf{G}_{\bullet}\right) \Rightarrow \pi_{s+t} \operatorname{diag} \mathbf{G}_{\bullet},
$$

is due to Quillen (cf. [Q2]).

The above definitions provide us with a functorial simplicial version of the derived composition of $\S 3.3$ : 
Definition 5.5. If $\mathbf{K} \in \mathcal{S}_{*}^{\mathrm{Kan}}$ is an $H$-simplicial set which is a Kan complex, one again has a retraction of simplicial sets $\bar{m}: F \mathbf{K} \rightarrow \mathbf{K}$, defined as in (3.2). Given a homomorphism of simplicial groups $f: F \mathbf{A} \rightarrow F \mathbf{B}$ and a map of simplicial sets $g: \mathbf{B} \rightarrow \mathbf{K}$, the composite $\bar{m} \circ F g \circ f: F \mathbf{A} \rightarrow \mathbf{K}$ will be denoted by $f \star g$.

Note that if $\tilde{f}: \Sigma \mathbf{A} \rightarrow \bar{W} F \mathbf{B}$ and $\bar{f}: \mathbf{A} \rightarrow F \mathbf{B}$ correspond to $f$ under (5.2), the composite $\bar{m} \circ F g \circ \bar{f}$ corresponds to $f \star g$, and represents the derived composition $[\bar{f}] \star[g]$ in $[\mathbf{A}, \mathbf{K}]_{\mathcal{S}_{*}} \cong[|\mathbf{A}|,|\mathbf{K}|]_{\mathcal{T}_{*}}$.

R e mark 5.6. The simplicial version of the $\star$ operation defined here is obviously functorial in the sense that $\left(e^{*} f\right) \star g=e^{*}(f \star g)$ for $e: F \mathbf{C} \rightarrow F \mathbf{A}$ in $\mathcal{G}$, and $f \star\left(g^{*} h\right)=(f \star g)^{*} h$ for any $H$-map $h:\langle\mathbf{K}, m\rangle \rightarrow\langle\mathbf{L}, n\rangle$ between fibrant $H$-simplicial sets which is strictly multiplicative (i.e., $n \circ(h \times h)=$ $h \circ m: \mathbf{K} \times \mathbf{K} \rightarrow \mathbf{L})$.

However, Proposition 3.8 is still valid only in the homotopy category, and this is in fact the obstruction to $\mathbf{K}$ being equivalent to a loop space:

THEOREM 5.7. If $\mathbf{K}$ is an $H$-group in $\mathcal{S}_{*}^{\text {Kan }}$ such that

$(*) \quad f \star(g \star h)=\left(f^{\#} g\right) \star h$ for all $f: F \mathbf{A} \rightarrow F \mathbf{B}$ and $g: F \mathbf{B} \rightarrow F \mathbf{C}$ in $\mathcal{G}$ and $h: \mathbf{C} \rightarrow \mathbf{K}$,

then $\mathbf{K}$ is $H$-homotopy equivalent to a simplicial group (and thus to a loop space); conversely, if $\mathbf{K} \in \mathcal{G}$ (in particular, if $\mathbf{K}=G \mathbf{L}$ for some $\mathbf{L} \in \mathcal{S}_{0}$ ), then $(*)$ holds.

Proof. Assume that $\mathbf{K}$ is an $H$-group in $\mathcal{S}_{*}^{\text {Kan }}$ satisfying $(*)$. We shall need a simplicial variant of Stover's construction of resolutions by spheres (Def. 4.6), so as in [Stv, §2], define a comonad $L: \mathcal{G} \rightarrow \mathcal{G}$ by

$$
L \mathbf{G}=\coprod_{k=0}^{\infty} \coprod_{\phi \in \mathrm{Hom}_{\mathcal{G}}\left(F S^{k}, G\right)} F \mathbf{S}_{\phi}^{k} \cup \coprod_{k=0}^{\infty} \coprod_{\Phi \in \mathrm{Hom}_{\mathcal{G}}\left(F \mathbf{e}^{k+1}, G\right)} F \mathbf{e}_{\Phi}^{k+1},
$$

where $F \mathbf{e}_{\Phi}^{k+1}$, the $\mathcal{G}$-disc indexed by $\Phi: F \mathbf{e}^{k+1} \rightarrow \mathbf{G}$, is attached to $F \mathbf{S}_{\phi}^{k}$, the $\mathcal{G}$-sphere indexed by $\phi=\left.\Phi\right|_{F \partial \mathbf{e}^{k+1}}$, by identifying $F \partial \mathbf{e}^{k+1}$ with $F \mathbf{S}^{k}$ (see $\S 5.1$ above). The coproduct here is just the (dimensionwise) free product of groups; the counit $\varepsilon: L \mathbf{G} \rightarrow \mathbf{G}$ is "evaluation of indices", and the comultiplication $\vartheta: L \mathbf{G} \hookrightarrow L^{2} \mathbf{G}$ is as in $\S 2.6$.

Now let

$$
\mathbf{W}=\bigvee_{k=1}^{\infty} \bigvee_{f \in \operatorname{Hom}_{\mathcal{G}}\left(S^{k}, K\right)} \mathbf{S}_{f}^{k} \cup \bigvee_{k=1}^{\infty} \bigvee_{F \in \operatorname{Hom}_{\mathcal{S}_{*}}\left(e^{k+1}, K\right)} \mathbf{e}_{F}^{k+1}
$$

(the analogue for $\mathcal{S}_{*}$ of $L \mathbf{G}$, with the corresponding identifications), and let $z: \mathbf{W} \rightarrow \mathbf{K}$ be the counit map. Then $z$ induces an epimorphism $z_{*}: \pi_{*} \mathbf{W} \rightarrow$ 
$\pi_{*} \mathbf{K}$ of $\Pi$-algebras. ( $\mathbf{K}$ is a Kan complex, but $\mathbf{W}$ is not, so we understand $\pi_{*} \mathbf{W}$ to be the corresponding free $\Pi$-algebra $\cong \pi_{*}|\mathbf{W}|-\operatorname{cf}$. $\left.\S 2.3\right)$.

Likewise, we have an epimorphism of $\Pi$-algebras $\widetilde{\zeta}: \pi_{*} \Sigma \mathbf{W} \rightarrow G_{*}$, where $G_{*}=\Omega^{-1} \pi_{*} \mathbf{K}$ is the delooping of $\pi_{*} \mathbf{K}$ - or equivalently, $\widetilde{z}_{*}: \pi_{*}^{H} F \mathbf{W} \rightarrow$ $\pi_{*}^{H} \mathbf{K}$, induced by $\widetilde{z}=\bar{m} \circ F z: F \mathbf{W} \rightarrow \mathbf{K}$ (cf. $\left.\S 5.5\right)$.

Let $\mathbf{M}_{n}=L^{n} F \mathbf{W}$ for $n=0,1, \ldots$, with face and degeneracy maps determined by the comonad structure maps $\varepsilon, \vartheta$ - except for $d_{n}: \mathbf{M}_{n} \rightarrow$ $\mathbf{M}_{n-1}$, defined by $d_{n}=L^{n-1} \bar{d}$, where $\bar{d}: L F \mathbf{W} \rightarrow F \mathbf{W}$, restricted to a summand $F \mathbf{A}_{\alpha}$ in $L F \mathbf{W}\left(\mathbf{A}=\mathbf{S}^{k}, \mathbf{e}^{k+1}\right)$, is an isomorphism onto $F \mathbf{A}_{\beta} \hookrightarrow$ $F \mathbf{W}$, where $\beta: \mathbf{A} \rightarrow \mathbf{K}$ is the composite $(\alpha \star z) \circ j_{A}$.

Because (*) holds exactly, we may verify that $\bar{d} \circ T \bar{d}=\bar{d} \circ T \varepsilon: \mathbf{M}_{2} \rightarrow \mathbf{M}_{0}$, so that $\mathbf{M}_{\bullet}$ is a simplicial object over $\mathcal{G}$. Moreover, the augmented simplicial $\Pi$-algebra $\pi_{*} \mathbf{M}_{\bullet} \stackrel{\widetilde{\zeta}}{\longrightarrow} G_{*}$ is acyclic, by a variant of [Stv, Prop. 2.6]. Thus the Quillen spectral sequence for $\mathbf{M}_{\bullet}$ (see (5.4)) has $E_{s, t}^{2}=0$ for $s>0$, and $E_{0, *}^{2} \cong G_{*}$, so it collapses, and $\pi_{*}^{H} \operatorname{diag} \mathbf{M}_{\bullet} \cong G_{*}=\pi_{*}^{H} \mathbf{K}$. Therefore, if we set $\mathbf{L}=\operatorname{diag} \bar{W} \mathbf{M}_{\bullet}$ (which is isomorphic to $\bar{W} \operatorname{diag} \mathbf{L}_{\bullet}$ ) we obtain a Kan complex $\mathbf{L}$ such that $\mathbf{K} \simeq G \mathbf{L}$-so $|\mathbf{K}| \simeq \Omega|\mathbf{L}|$.

The converse is clear, since if $\mathbf{K} \in \mathcal{G}$ then $j_{A}: \mathbf{A} \rightarrow F \mathbf{A}$ induces a oneto-one correspondence between maps $f: \mathbf{A} \rightarrow \mathbf{K}$ in $\mathcal{S}_{*}$ and homomorphisms $\varphi: F \mathbf{A} \rightarrow \mathbf{K}$ in $\mathcal{G}$, by the universal property of $F$.

6. Rectifying simplicial spaces. Theorem 5.7 suggests a way to determine whether an $H$-group $\mathbf{X}$ is equivalent to a loop space. Note that in fact we need only verify that $5.7(*)$ holds for $\mathbf{A}, \mathbf{B}$, and $\mathbf{C}$ in $\mathcal{S}_{*}$ which are homotopy equivalent to wedges of spheres. We now suggest a universal collection of examples which may be used for this purpose, organized into one (very large!) simplicial diagram. First, some definitions:

Definition 6.1. A simplicial space up-to-homotopy is a diagram ${ }^{h} \mathbf{W}$. over $\mathcal{T}_{*}$ consisting of a sequence of spaces $\mathbf{W}_{0}, \mathbf{W}_{1}, \ldots$, together with face and degeneracy maps $d_{i}: \mathbf{W}_{n} \rightarrow \mathbf{W}_{n-1}$ and $s_{j}: \mathbf{W}_{n} \rightarrow \mathbf{W}_{n+1}(0 \leq i, j$ $\leq n$ ), satisfying the simplicial identities only up to homotopy.

Note that such a diagram constitutes an ordinary simplicial object over $h o \mathcal{T}_{*}$, so we can apply the functor $\pi_{*}: \mathcal{T}_{*} \rightarrow \Pi$-Alg to ${ }^{h} \mathbf{W}$. to obtain an (honest) simplicial $\Pi$-algebra $\pi_{*}\left({ }^{h} \mathbf{W}_{\bullet}\right) \in s \Pi$-Alg. Similarly for a $\Delta$ simplicial space up-to-homotopy ${ }^{h} \mathbf{W}_{\bullet}^{\Delta}$.

Remark 6.2. Note that diagrams denoted by $\mathbf{W}_{\bullet},{ }^{h} \mathbf{W}_{\bullet}, \mathbf{W}_{\bullet}^{\Delta}$, and ${ }^{h} \mathbf{W}_{\bullet}^{\Delta}$ each consist of a sequence of spaces $\mathbf{W}_{0}, \mathbf{W}_{1}, \ldots$; they differ in the maps with which they are equipped, and whether the identities which these maps are required to satisfy must hold in $\mathcal{T}_{*}$ or only in $h o \mathcal{T}_{*}$. 
Definition 6.3. An (ordinary) simplicial space $\mathbf{V} \bullet \in s \mathcal{T}_{*}$ is called a rectification of a simplicial space up-to-homotopy ${ }^{h} \mathbf{W}_{\text {. if }} \mathbf{V}_{n} \simeq \mathbf{W}_{n}$ for each $n \geq 0$, and the face and degeneracy maps of $\mathbf{V}$. are homotopic to the corresponding maps of ${ }^{h} \mathbf{W}$. (see [DKS, §2.2], e.g., for a more precise definition). For our purposes all we require is that $\pi_{*} \mathbf{V} \bullet$ be isomorphic (as a simplicial $\Pi$-algebra) to $\pi_{*}\left({ }^{h} \mathbf{W}_{\bullet}\right)$. Similarly for rectification of $\Delta$-simplicial spaces, and $\left(\Delta\right.$-)simplicial objects in $h o \mathcal{S}_{*}^{\text {Kan }}$ or $h o \mathcal{G}$.

6.4. A $\Delta$-simplicial space up-to-homotopy. Given an $H$-group $\mathbf{X}$, we wish to determine whether it is a loop space, up to homotopy. We start by choosing some free simplicial $\Pi$-algebra $A_{*}$ resolving $G_{*}=\Omega^{-1} \pi_{*} \mathbf{X}$. By Remark 2.4, the free simplicial $\Pi$-algebra $A_{*}$ corresponds to a simplicial object over the homotopy category, unique up to isomorphism (in $\left.h o \mathcal{T}_{*}\right)$, with each space homotopy equivalent to a wedge of spheres. Therefore, it may be represented by a simplicial space up-to-homotopy ${ }^{h} \mathbf{W}_{\bullet}$, with $\pi_{*}\left({ }^{h} \mathbf{W}_{\bullet}\right) \cong A_{* \bullet}(\S 6.1)$. We denote its underlying $\Delta$-simplicial space up-to-homotopy by ${ }^{h} \mathbf{W}_{\bullet}^{\Delta}$.

In light of Theorem 5.7 it would perhaps be more natural to consider the corresponding simplicial object up-to-homotopy over $\mathcal{G}$, or $\mathcal{S}_{*}$, but given the equivalence of homotopy categories $h o \mathcal{T}_{*} \cong h o \mathcal{G} \cong h o \mathcal{S}_{*}$, we prefer to work in the more familiar topological category.

Now ${ }^{h} \mathbf{W}$. may be rectified if and only if it can be made $\infty$-homotopy commutative - that is, if and only if one can find a sequence of homotopies for the simplicial identities among the face and degeneracy maps, and then homotopies between these, and so on (cf. [BV, Corollary 4.21 \& Theorem 4.49]). An obstruction theory for this was described in [B13], and we briefly recall the main ideas here, mainly because we wish to present a technical simplification which eliminates the need for $[\mathrm{Bl} 3, \S 6]$ : as we shall see below, it suffices to rectify ${ }^{h} \mathbf{W}_{\bullet}^{\Delta}$; so we describe an obstruction theory for the rectification of $\Delta$-simplicial spaces up-to-homotopy. For this, we need some definitions from $[\mathrm{Bl} 3, \S 5]$ :

Definition 6.5. The $k$-dimensional permutohedron $P_{k}$ is defined to be the convex hull in $\mathbb{R}^{k+1}$ of the $(k+1)$ ! points $(\sigma(1), \sigma(2), \ldots, \sigma(k+1)) \in$ $\mathbb{R}^{k+1}$, indexed by permutations $\sigma \in \Sigma_{k+1}$ (cf. [Zi, 0.10]). Its boundary is denoted by $\partial P_{k}$.

For $n \geq 0$ and any morphism $\delta: \mathbf{n}+\mathbf{1} \rightarrow \mathbf{n}-\mathbf{k}$ in $\Delta_{\partial}^{\text {op }}$ (see $\S 1.2$ above), we may label the vertices of $P_{k}$ by all possible ways of writing $\delta$ as a composite of face maps (cf. [Bl3, Lemma 4.7]), and one can similarly interpret the faces of $P_{k}$. We shall write $P_{k}(\delta)$ for $P_{k}$ so labelled (thought of as an abstract combinatorial polyhedron). 
Definition 6.6. Let ${ }^{h} \mathbf{W}_{\bullet}^{\Delta}$ be a $\Delta$-simplicial space up-to-homotopy, and $\delta: \mathbf{n}+\mathbf{1} \rightarrow \mathbf{n}-\mathbf{k}$ some morphism in $\Delta_{\partial}^{\mathrm{op}}$. We denote by $C(\delta)$ the collection of all proper factors of $\delta$ - that is, $\gamma \in C(\delta) \Leftrightarrow \gamma^{\prime} \circ \gamma \circ \gamma^{\prime \prime}=\delta$ and $\gamma^{\prime}, \gamma^{\prime \prime}$ are not both $i d$.

A compatible collection for $C(\delta)$ and ${ }^{h} \mathbf{W}_{\bullet}^{\Delta}$ is a set $\left\{g^{\gamma}\right\}_{\gamma \in C(\delta)}$ of maps $g^{\gamma}: P_{n-k} \ltimes \mathbf{W}_{n} \rightarrow \mathbf{W}_{k-1}$, one for each $\gamma=\left[\left(i_{k}, \ldots, i_{n}\right)\right] \in C(\delta)$, such that for any partition $\left\langle i_{k}, \ldots, i_{\ell_{1}}\left|i_{\ell_{1}+1}, \ldots, i_{\ell_{2}}\right| \ldots \mid i_{\ell_{r-1}+1}, \ldots, i_{n}\right\rangle$ of $i_{k}, \ldots, i_{n}$ into $r$ blocks (where $\gamma=d_{i_{n}} \circ \ldots \circ d_{i_{k}}$ in $\Delta_{\partial}^{\mathrm{op}}$ ), with $\gamma_{1}=d_{i_{\ell_{1}}} \circ$ $\ldots \circ d_{i_{k}}, \ldots, \gamma_{r}=d_{i_{n}} \circ \ldots \circ d_{i_{\ell_{r-1}+1}}$, and we set $P:=P_{\ell_{1}-k-1}\left(\gamma_{1}\right) \times$ $P_{\ell_{2}-\ell_{1}}\left(\gamma_{2}\right) \ldots P_{n-\ell_{r-1}}\left(\gamma_{r}\right)$, then we require that $\left.g^{\gamma}\right|_{P \ltimes Y n}$ be the composite of the corresponding maps $g^{\gamma_{i}}$, in the obvious sense. We further require that if $\gamma=\left[i_{j}\right]$, then $g^{\gamma}$ must be in the prescribed homotopy class of $\left[d_{i_{j}}\right] \in$ $\left[\mathbf{W}_{j+1}, \mathbf{W}_{j}\right]$.

We shall be interested in such compatible collections only up to the obvious homotopy relation. Note that for any $\delta: \mathbf{n}+\mathbf{1} \rightarrow \mathbf{n}-\mathbf{k}$ in $\Delta_{\partial}^{\text {op }}$, any compatible collection $\left\{g^{\gamma}\right\}_{\gamma \in C(\delta)}$ induces a map $f=f^{\delta}: \partial P_{k} \ltimes \mathbf{W}_{n+1} \rightarrow$ $\mathbf{W}_{n-k}$, and compatibly homotopic collections induce homotopic maps.

Definition 6.7. Given ${ }^{h} \mathbf{W}_{\bullet}^{\Delta}$ as in $\S 6.4$, for each $k \geq 2$ and $\delta: \mathbf{n}+\mathbf{1} \rightarrow$ $\mathbf{n}-\mathbf{k} \in \Delta_{\partial}^{\mathrm{op}}$, the $k$ th order homotopy operation (associated with ${ }^{h} \mathbf{W}_{\bullet}^{\Delta}$ and $\delta$ ) is a subset $\langle\langle\delta\rangle\rangle$ of the track group $\left[\Sigma^{k-1} \mathbf{W}_{n+1}, \mathbf{W}_{n-k}\right]$, defined as follows:

Let $S \subseteq\left[\partial P_{k} \ltimes \mathbf{W}_{n+1}, \mathbf{W}_{n-k}\right]$ be the set of homotopy classes of maps $f=f^{\delta}: \partial P_{k}(\delta) \ltimes \mathbf{W}_{n+1} \rightarrow \mathbf{W}_{n-k}$ which are induced as above by some compatible collection $\left\{g^{\gamma}\right\}_{\gamma \in C(\delta)}$. Choose a splitting

$$
\partial P_{k}(\delta) \ltimes \mathbf{W}_{n+1} \cong \mathbf{S}^{k-1} \ltimes \mathbf{W}_{n+1} \simeq \mathbf{S}^{k-1} \wedge \mathbf{W}_{n+1} \vee \mathbf{W}_{n+1},
$$

and let $\langle\langle\delta\rangle\rangle \subseteq\left[\Sigma^{k-1} \mathbf{W}_{n+1}, \mathbf{W}_{n-k}\right]$ be the image under the resulting projection of the subset $S \subseteq\left[\partial P_{k} \ltimes \mathbf{W}_{n+1}, \mathbf{W}_{n-k}\right]$.

6.8. Coherent vanishing. It is clearly a necessary condition in order for the subset $\langle\langle\delta\rangle\rangle$ to be non-empty that all the lower order operations (for $\gamma \in$ $C(\delta)$ ) vanish-i.e., contain the null class; a sufficient condition is that they do so coherently, in the sense of $[\mathrm{Bl} 3, \S 5.7]$. Again one may define a collection of higher homotopy operations in various track groups $\left[\Sigma \mathbf{W}_{m}, \mathbf{W}_{m-\ell}\right]$, whose vanishing guarantees the coherence of a given collection of maps (see [Bl3, $\S 5.9])$. One then has

Proposition 6.9 (see Theorem 6.12 of [Bl3]). Given a $\Delta$-simplicial space up-to-homotopy ${ }^{h} \mathbf{W}_{\bullet}^{\Delta}$, it may be rectified to a strict $\Delta$-simplicial space $\mathbf{V}_{\bullet}^{\Delta}$ if and only if all the sequence of higher homotopy operations defined above vanish coherently.

6.10. Adding degeneracies. Now assume given a $\Delta$-simplicial space $\mathbf{W}_{\bullet}^{\Delta} \in$ $\Delta \mathcal{T}_{*}$, to which we wish to add degeneracies, in order to obtain a full sim- 
plicial space $\mathbf{W}_{\bullet}$. Note that because the original $\Delta$-simplicial space up-tohomotopy ${ }^{h} \mathbf{W}_{\bullet}^{\Delta}$ of $\S 6.4$ above was obtained from the simplicial $\Pi$-algebra $A_{* \bullet}$, in the case of interest to us $\mathbf{W}_{\bullet}^{\Delta}$ is already equipped with degeneracy maps - but these satisfy the simplicial identities only up to homotopy!

In this situation, a similar obstruction theory was defined in $[\mathrm{Bl} 3, \S 6]$ for rectifying the degeneracies; but it was conjectured there that this theory is actually unnecessary ([Bl3, Conj. 6.9]). We now show this is in fact correct.

Definition 6.11. Given a $\Delta$-simplicial space $\mathbf{V}_{\bullet}^{\Delta}$, its $n$th matching space $M_{n} \mathbf{V}_{\bullet}^{\Delta}$ is defined to be the limit

$$
\begin{aligned}
M_{n} \mathbf{V}_{\bullet}^{\Delta}:=\left\{\left(x_{0}, \ldots, x_{n}\right) \in\left(\mathbf{V}_{n-1}\right)^{n+1} \mid d_{i} x_{j}=\right. & d_{j-1} x_{i} \\
& \text { for all } 0 \leq i<j \leq n\} .
\end{aligned}
$$

The map $\delta_{n}: \mathbf{V}_{n} \rightarrow M_{n} \mathbf{V}_{\bullet}^{\Delta}$ is defined by $\delta_{n}(x)=\left(d_{0} x, \ldots, d_{n} x\right)$. (See [Hi, XVII, 87.17], and compare [BK, X, §4.5].)

Definition 6.12. A $\Delta$-simplicial space $\mathbf{V}_{\bullet}^{\Delta}$ is called Kan if for each $n \geq 1$ the map $\delta_{n}: \mathbf{V}_{n} \rightarrow M_{n} \mathbf{V}_{\bullet}^{\Delta}$ is a fibration. (See [Hi, XVII, 88.2] or [DHK, XII, §54], where this is called a Reedy fibrant object.)

Lemma 6.13. For any $\Delta$-simplicial space $\mathbf{X}_{\bullet}^{\Delta}$, there is a Kan $\Delta$-simplicial space $\mathbf{V}_{\bullet}^{\Delta}$ and a map of $\Delta$-simplicial spaces $f_{\bullet}: \mathbf{X}_{\bullet}^{\Delta} \rightarrow \mathbf{V}_{\bullet}^{\Delta}$ such that each $f_{n}$ is a homotopy equivalence.

Proof. This follows from the existence of the so-called Reedy model category structure on $\Delta \mathcal{T}_{*}$ (see [Hi, XVII, Thm. 88.3]); $\mathbf{V}_{\bullet}^{\Delta}$ may be constructed directly from $\mathbf{X}_{\bullet}^{\Delta}$ by successively changing the maps $\delta_{n}$ into fibrations as in [W, I, (7.30)].

Thus the proof of [Bl3, Conj. 6.9] follows from:

Proposition 6.14 (compare Theorem 5.7 of [RS]). If $\mathbf{V}_{\bullet}^{\Delta}$ is a Kan $\Delta$ simplicial space which rectifies the $\Delta$-simplicial space up-to-homotopy ${ }^{h} \mathbf{W}_{\bullet}^{\Delta}$ of $\S 6.4$, then one can define degeneracy maps on $\mathbf{V}_{\bullet}^{\Delta}$ making it into a full simplicial space.

Proof. Using the singular functor $S: \mathcal{T}_{*} \rightarrow \mathcal{S}_{*}^{\text {Kan }}$ we may work with simplicial sets, rather than topological spaces; the maps $\delta_{n}: \mathbf{V}_{n} \rightarrow M_{n} \mathbf{V}_{\bullet}^{\Delta}$ are now assumed to be Kan fibrations in $\mathcal{S}_{*}$.

By induction on $n \geq 0$ we assume that degeneracy maps $s_{j}: \mathbf{V}_{k} \rightarrow \mathbf{V}_{k+1}$ have been chosen for all $0 \leq j \leq k<n$, satisfying all relevant simplicial identities.

Let $\Sigma$ denote the subcategory of $\Delta^{\mathrm{op}}$ with the same objects as $\Delta^{\mathrm{op}}$, but only the degeneracies as morphisms. For each $k \geq 0$, let $\Sigma / \mathbf{k}$ denote the "over category" of $\mathbf{k}$. By assumption $\mathbf{V}_{\bullet}^{\Delta}$, together with the existing degeneracies, defines a functor $V: \Sigma / \mathbf{n}-\mathbf{1} \rightarrow \mathcal{S}_{*}$. Denote its colimit by 
$L_{n}$; this may be thought of as the sub-simplicial set of $\mathbf{V}_{n}$ consisting of the degenerate simplices; i.e., $\bigcup_{j=0}^{n-1} \operatorname{Im}\left(s_{j}\right)$. We also have an associated "free" functor $\Sigma / \mathbf{m} \rightarrow \mathcal{S}_{*}$ for each $m \geq 0$ (with the same values on objects as $V$, but all morphisms trivial). Its colimit, denoted by $D_{m}$, is the coproduct (i.e., wedge) over $\operatorname{Obj}(\Sigma / \mathbf{m})$ of the spaces $\mathbf{V}_{k}(0 \leq k<m)$, indexed by all possible iterated degeneracies $s_{i_{1}} \circ \ldots \circ s_{i_{m-k}}: \mathbf{k} \rightarrow \mathbf{m}$. This comes equipped with structure maps $e_{j}^{m-1}: \mathbf{V}_{m-1} \rightarrow D_{m}(0 \leq j<m)$. See [Mal, p. 95] or [Bl1, §4.5.1] for an explicit description. For example, $L_{0}=$ $D_{0}=*, L_{1}=D_{1} \cong \mathbf{V}_{0}$, but $D_{2}=\left(\mathbf{V}_{1}\right)_{s_{0}} \vee\left(\mathbf{V}_{0}\right)_{s_{1} s_{0}} \vee\left(\mathbf{V}_{1}\right)_{s_{1}}$, while $L_{2}=\left(\mathbf{V}_{1}\right)_{s_{0}} \amalg_{\left(\mathbf{V}_{0}\right)_{s_{1} s_{0}}}\left(\mathbf{V}_{1}\right)_{s_{1}}$ (the pushout).

In fact, if we define $\mathbf{Y}_{\bullet} \in s \mathcal{S}_{*}$ by $\mathbf{Y}_{n}:=\mathbf{V}_{n} \vee D_{n}$, with the obvious degeneracies (defined by the structure maps $e_{j}^{m}$ ) and face maps (induced from those of $\mathbf{V}_{\bullet}^{\Delta}$ via the simplicial identities), then $F\left(\mathbf{V}_{\bullet}^{\Delta}\right):=\mathbf{Y}_{\bullet}$ defines a functor $F: \Delta \mathcal{S}_{*} \rightarrow s \mathcal{S}_{*}$ which is left adjoint to the forgetful functor $U: s \mathcal{S}_{*} \rightarrow \Delta \mathcal{S}_{*}$, and there is a natural inclusion $\iota: \mathbf{V}_{\bullet}^{\Delta} \rightarrow U F \mathbf{V}_{\bullet}^{\Delta}$.

Note that the degeneracies up-to-homotopy $s_{j}^{\prime}: \mathbf{V}_{n} \rightarrow \mathbf{V}_{n}$, which exist because $\mathbf{V}_{\bullet}^{\Delta}$ rectifies $U\left({ }^{h} \mathbf{W}_{\bullet}\right)$ (where $\pi_{*}\left({ }^{h} \mathbf{W}_{\bullet}\right) \cong A_{*}$ as simplicial $\Pi$ algebras), define a map $\sigma_{n+1}^{\prime}: D_{n+1} \rightarrow V_{n+1}$.

Our objective is to define inductively a retraction $\sigma: U F \mathbf{V}_{\bullet}^{\Delta} \rightarrow \mathbf{V}_{\bullet}^{\Delta}$ in $\Delta \mathcal{S}_{*}$, starting with $\sigma_{0}=\operatorname{id}_{\mathbf{V}_{0}}$, such that $\sigma_{n} \sim \sigma_{n}^{\prime}$ for all $n$. This map $\sigma$ must commute with the degeneracies defined so far: that is, at the $n$th stage we must choose a map $\sigma_{n+1}: D_{n+1} \rightarrow \mathbf{V}_{n+1}$ homotopic to $\sigma_{n+1}^{\prime}$, and then define $s_{j}^{n}: \mathbf{V}_{n} \rightarrow \mathbf{V}_{n+1}$ by

$$
s_{j}^{n}:=\sigma_{n+1} \circ e_{j}^{n} \circ \iota_{n} .
$$

Moreover, together with the face maps of $\mathbf{V}_{\bullet}^{\Delta}$, the degeneracies chosen so far determine a map $\varrho_{n}: D_{n+1} \mathbf{V}_{\bullet}^{\Delta} \rightarrow M_{n+1} \mathbf{V}_{\bullet}^{\Delta}$, by the universal properties of the limit and colimit; the simplicial identities in ${ }^{h} \mathbf{W}$. guarantee that $\delta_{n+1} \circ \sigma_{n+1}^{\prime} \sim \varrho_{n+1}$. Note than in order for the simplicial identities $d_{i} s_{j}=$ $s_{j-1} d_{i}($ for $i<j), d_{j} s_{j}=d_{j+1} s_{j}=i d$, and $d_{i} s_{j}=s_{j} d_{i-1}($ for $i>j+1)$ to be satisfied, it suffices that

$$
\delta_{n+1} \circ \sigma_{n+1}=\varrho_{n+1},
$$

that is, $\sigma_{n+1}$ must be a lift for the given map $\varrho_{n+1}$. On the other hand, in order that $s_{j} s_{i}=s_{i+1} s_{j}$ hold for all $j \leq i$, it suffices to have

$$
\sigma_{n+1} \circ e_{j}^{n}=\sigma_{n+1} \circ e_{j}^{n} \circ \iota_{n} \circ \sigma_{n} \quad \text { for all } 0 \leq j \leq n
$$

(where $\iota_{n}: \mathbf{V}_{n} \hookrightarrow\left(U F \mathbf{V}_{\bullet}^{\Delta}\right)_{n}$ is the inclusion).

Now $D_{n+1}$ has a wedge summand $\bar{D}_{n+1}$ such that $D_{n+1}=\bar{D}_{n+1} \vee$ $\bigvee_{j=0}^{n}\left(\mathbf{V}_{n}\right)_{s_{j}}$, and $\sigma_{n+1}^{\prime}: D_{n+1} \rightarrow \mathbf{V}_{n+1}$ thus defines a map $\bar{\sigma}_{n+1}^{\prime}=$ $\left.\sigma_{n+1}^{\prime}\right|_{\bar{D}_{n+1}}: \bar{D}_{n+1} \rightarrow \mathbf{V}_{n+1}$. Since $\delta_{n+1}$ is a fibration, one may use the homo- 
topy lifting property to obtain a map $\bar{\sigma}_{n+1} \sim \bar{\sigma}_{n+1}^{\prime}$ such that $\delta_{n+1} \circ \bar{\sigma}_{n+1}=$ $\left.\varrho_{n+1}\right|_{\bar{D}_{n+1}}$.

Note that $L_{n}=\operatorname{Im}\left(\sigma_{n}\right)$, by induction, so (6.17) for $n-1$ and (6.15) imply that, for each $0 \leq j \leq n$, the map $\bar{\sigma}_{n+1} \circ e_{j}^{n}: D_{n} \rightarrow \mathbf{V}_{n+1}$ induces a $\operatorname{map} g_{j}: L_{n} \rightarrow \mathbf{V}_{n+1}$.

Because $A_{* \bullet}$ is a free simplicial $\Pi$-algebra (Def. 4.3) and $\pi_{*}\left({ }^{h} \mathbf{W}_{\bullet}\right) \cong A_{* \bullet}$, Lemma 2.5 guarantees that there is a $\mathbf{Z}_{n} \in \mathcal{S}_{*}$, weakly equivalent to a wedge of spheres, and a map $f_{n}: \mathbf{Z}_{n} \rightarrow \mathbf{V}_{n}$ which, together with the inclusion $h_{n}: L_{n} \hookrightarrow \mathbf{V}_{n}$, induces a weak equivalence of simplicial sets $\left(h_{n}, f_{n}\right): L_{n} \vee \mathbf{Z}_{n} \stackrel{\simeq}{\rightarrow} \mathbf{V}_{n}$. Since $h_{n}$ is a cofibration, using a minimal complex for $\mathbf{Z}_{n}$ (see [Ma1, §9]) we may assume that $\left(h_{n}, f_{n}\right)$ is a trivial cofibration in $\mathcal{S}_{*}$ (cf. [Q1, II, 3.14]). Again the fact that $\delta_{n+1}$ is a fibration implies that there exists a lifting $\alpha_{j}: \mathbf{Z}_{n} \rightarrow \mathbf{V}_{n+1}$ for $\left.\varrho_{n+1}\right|_{\left(\mathbf{V}_{n}\right)_{s_{j}}} \circ f_{n}: \mathbf{Z}_{n} \rightarrow M_{n+1} \mathbf{V}_{\bullet}^{\Delta}$. Thus the left lifting property (cf. [Q1, I, 5.1]) for the solid commutative square

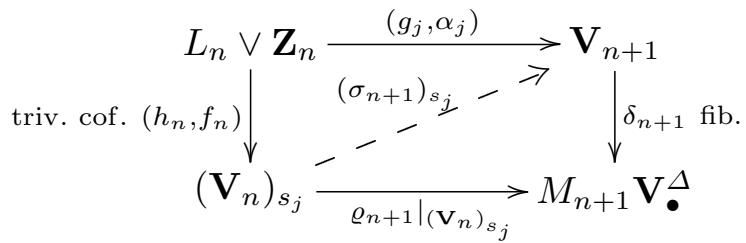

guarantees the existence of a dashed lifting $\left(\sigma_{n+1}\right)_{s_{j}}:\left(\mathbf{V}_{n}\right)_{s_{j}} \rightarrow \mathbf{V}_{n+1}$ for $\left.\varrho_{n+1}\right|_{\left(\mathbf{V}_{n}\right)_{s_{j}}}$, and these liftings, for various $j$, together with $\bar{\sigma}_{n+1}$, define $\sigma_{n+1}: D_{n+1} \rightarrow \mathbf{V}_{n+1}$ satisfying (6.17) (and of course (6.16)), as required. (6.15) then defines $s_{j}: \mathbf{V}_{n} \rightarrow \mathbf{V}_{n+1}$ for all $0 \leq j \leq n$, completing the induction.

Corollary 6.18. If $\mathbf{V}_{\bullet}^{\Delta}$ is a $\Delta$-simplicial space such that $\pi_{*} \mathbf{V}_{\bullet}^{\Delta}$ is a free simplicial $\Pi$-algebra (Def. 4.3), then there is a spectral sequence with $E_{s, t}^{2}=\pi_{s}\left(\pi_{t} \mathbf{V}_{\bullet}^{\Delta}\right) \Rightarrow \pi_{s+t}\left\|\mathbf{V}_{\bullet}^{\Delta}\right\|$.

Proof. See $\S 4.4$ and 4.5, noting that the definition of the homotopy groups of a simplicial group is also valid for a $\Delta$-simplicial group (see [Mal, $\S 17]$ ), and that in the proof of Proposition 6.14 we did not use the fact that $A_{* \bullet}$ was a resolution of $G_{*}$.

If the higher homotopy operations described in $\S 6.7$ vanish coherently, then the $\Delta$-simplicial space up-to-homotopy ${ }^{h} \mathbf{W}_{\bullet}^{\Delta}$ of $\S 6.4$ may be rectified to a strict $\Delta$-simplicial space $\mathbf{W}_{\bullet}^{\Delta}$, which may in turn be replaced by a Kan $\Delta$-simplicial space $\mathbf{V}_{\bullet}^{\Delta}$ using Lemma 6.13 , with $\pi_{*} \mathbf{V}_{\bullet}^{\Delta} \cong A_{* \bullet}$. The spectral sequence of Corollary 6.18 then implies that $\mathbf{Y}:=\left\|\mathbf{V}_{\bullet}^{\Delta}\right\|$ satisfies $\pi_{*} \mathbf{Y} \cong G_{*}=\Omega^{-1} \pi_{*} \mathbf{X}$. We have thus realized the algebraic delooping of $\pi_{*} \mathbf{X}$ by a space $\mathbf{Y}$. 
Remark 6.19. As in any obstruction theory, if one of the homotopy operations in question does not vanish (or if there is a non-vanishing obstruction to coherence, as in $\S 6.8$ ), one must backtrack, changing choices made at previous stages. On the face of it, if all such choices show that the $\Delta$-simplicial space up-to-homotopy ${ }^{h} \mathbf{W}_{\bullet}^{\Delta}$ cannot be rectified, we must then try other choices for the resolution $A_{* \bullet} \rightarrow G_{*}$. However, we conjecture that in fact if one free simplicial $\Pi$-algebra resolution of $G_{*}=\Omega^{-1} \pi_{*} \mathbf{X}$ is realizable, then any resolution is realizable (so that any obstruction to rectifying ${ }^{h} \mathbf{W}$. shows that $\mathbf{X}$ is not a loop space).

6.20. Realizing $\Pi$-algebra morphisms. It remains to ascertain that the space $\mathbf{Y}$ which realizes $G_{*}$ is in fact a delooping of $\mathbf{X}$. In other words, we have an abstract $\Pi$-algebra isomorphism $\phi: \pi_{*} \Omega \mathbf{Y} \cong \pi_{*} \mathbf{X}$ (cf. Corollary 3.9 ), which we wish to realize by a map of spaces $f: \Omega \mathbf{Y} \rightarrow \mathbf{X}$. Now, there is an obstruction theory for the realization of $\Pi$-algebra morphisms, simpler than but similar in spirit to that described above, which we briefly recapitulate. For the details, see [Bl3, §7], as simplified in [Bl4, §4.9] (and see $[\mathrm{B} 15, \S 4]$ for an algebraic version).

We start with some $\Delta$-simplicial resolution of $\Omega \mathbf{Y}$ by wedges of spheresi.e., an augmented $\Delta$-simplicial space $\mathbf{V}_{\bullet}^{\Delta} \stackrel{\varepsilon}{\rightarrow} \Omega \mathbf{Y}$ such that $\pi_{*} \mathbf{V}_{\bullet}^{\Delta}$ is a $\Delta$ simplicial $\Pi$-algebra resolution of $\pi_{*} \Omega \mathbf{Y} \cong \pi_{*} \mathbf{X}$, and each $\mathbf{V}_{n}$ is homotopy equivalent to a wedge of spheres (see $[\operatorname{Stv}, \S 1]$ ). The spectral sequence of Corollary 6.18 then implies that $\left|\mathbf{V}_{\bullet}^{\Delta}\right| \simeq \Omega \mathbf{Y}$.

By Fact 2.4, we can realize $\varepsilon: \pi_{*} \mathbf{V}_{0} \rightarrow \pi_{*} \mathbf{X}$ by a map $e_{0}: \mathbf{V}_{0} \rightarrow \mathbf{X}$, and then define $e_{n}: \mathbf{V}_{n} \rightarrow \mathbf{X}$ by $e_{n}:=e_{n-1} \circ d_{n}$ for $n>0$. By the simplicial identities for $\pi_{*} \mathbf{V}_{\bullet}^{\Delta} \rightarrow \pi_{*} \Omega \mathbf{Y}$, we know $\pi_{*}\left(e_{n}\right)=\pi_{*}\left(e_{n-1}\right) \circ d_{i}$, so that $e_{n} \sim e_{n-1} \circ d_{i}$ for all $0 \leq i \leq n$. If we can make this hold on the nose, rather than just up to homotopy, then $\mathbf{V}_{\bullet}^{\Delta} \stackrel{e_{0}}{\rightarrow} \mathbf{X}$ is also a (strict) augmented $\Delta$-simplicial space, so the spectral sequence of Corollary 6.18 now implies that $\left|\mathbf{V}_{\bullet}^{\Delta}\right| \simeq \mathbf{X}$, and thus $\Omega \mathbf{Y} \simeq \mathbf{X}$. This is where the appropriate higher homotopy operations (defined as follows) come in:

Definition 6.21. Let $D[n]$ denote the standard simplicial $n$-simplex, together with an indexing of its non-degenerate $k$-dimensional faces $D[k]^{(\gamma)}$ by the composite face maps $\gamma=d_{i_{n-k}} \circ \ldots \circ d_{i_{n}}: \mathbf{n} \rightarrow \mathbf{k}-\mathbf{1}$ in $\Delta^{\mathrm{op}}$. Its $(n-1)$-skeleton, which is a simplicial $(n-1)$-sphere, is denoted by $\partial D[n]$. We choose once and for all a fixed isomorphism $\varphi^{(\gamma)}: D[k]^{(\gamma)} \rightarrow D[k]$ for each face $D[k]^{(\gamma)}$ of $D[n]$.

Definition 6.22. Given $\mathbf{V}_{\bullet}^{\Delta}$ as above, for each $n \in \mathbb{N}$ we define a $\partial D[n]$ compatible sequence to be a sequence of maps $\left\{h_{k}: D[k] \ltimes \mathbf{V}_{k} \rightarrow \mathbf{X}\right\}_{k=0}^{n-1}$ such that $h_{0}=e_{0}$, and for any iterated face maps $\delta=d_{i_{j+1}} \circ \ldots \circ d_{i_{n}}$ and 
$\gamma=d_{i_{j}} \circ \delta(0 \leq j<n)$ we have $h_{j} \circ\left(\mathrm{id} \ltimes d_{i_{j}}\right)=h_{j+1} \circ\left(\iota_{\delta}^{\gamma} \ltimes \mathrm{id}\right)$ on $D[j] \ltimes \mathbf{V}_{j+1}$, where $\iota_{\delta}^{\gamma}:=\varphi^{\delta} \circ \iota \circ\left(\varphi^{\gamma}\right)^{-1}$, and $\iota: D[j]^{(\gamma)} \rightarrow D[j+1]^{(\delta)}$ is the inclusion.

Any such $\partial D[n]$-compatible sequence $\left\{h_{k}: D[k] \ltimes \mathbf{V}_{k} \rightarrow \mathbf{X}\right\}_{k=0}^{n-1}$ induces a map $\bar{h}: \partial D[n] \ltimes \mathbf{V}_{n} \rightarrow \mathbf{X}$, defined on the "faces" $D[n-1]^{\left(d_{i}\right)} \ltimes \mathbf{V}_{n}$ by $\left.\bar{h}\right|_{D[n-1]^{\left(d_{i}\right)} \ltimes \mathbf{V}_{n}}=h_{n-1} \circ\left(\mathrm{id} \ltimes d_{i}\right)$, and for each $n \geq 2$, the $n$th order homotopy operation (associated with $\mathbf{V}_{\bullet}^{\Delta}$ ) is a subset $\langle\langle n\rangle\rangle$ of the track group $\left[\Sigma^{n-1} \mathbf{V}_{n}, \mathbf{X}\right]$ defined analogously to $\S 6.7$.

Again as in $\S 6.8$, the coherent vanishing of all the operations $\{\langle n\rangle\rangle\}_{n=2}^{\infty}$ is a necessary and sufficient condition for $\Omega \mathbf{Y} \simeq \mathbf{X}$.

R e mark 6.23. We observe that if we choose to work in the category $\mathcal{S}_{*}^{\text {Kan }}$, rather than $\mathcal{T}_{*}$, we replace $\Omega \mathbf{Y}$ by $G(S \mathbf{Y})$, and we may then use the resolution $\mathbf{M}_{\bullet} \rightarrow G(S \mathbf{Y})$ of Theorem 5.7 (or rather, $\mathbf{M}_{\bullet}^{\Delta} \rightarrow G(S \mathbf{Y})$ ) instead of $\mathbf{V}_{\bullet}^{\Delta} \rightarrow \Omega \mathbf{Y}$. The obstruction theory that arises is of course equivalent to the one we just sketched, but it fits directly into the philosophy of Section 5, since our theory implies that $\mathbf{X}$ is a loop space if and only if the $H$-group augmentation up-to-homotopy from the given bisimplicial group $\mathbf{M}_{\bullet}$ to $S \mathbf{X}$ can be rectified.

We summarize the results of this section in

TheOREM 6.24. An H-group $\mathbf{X}$ is $H$-equivalent to a loop space if and only if the collection of higher homotopy operations defined in $\S 6.7$ and $\S 6.20$ above (taking values in homotopy groups of spheres or in $\pi_{*} \mathbf{X}$, respectively) vanish coherently.

Re mark 6.25. The obstruction theory described here is not applicable as such to the related question of the existence of $A_{n}$-structures on an $H$ space $\mathbf{X}$ (cf. [St1, §2]). An alternative approach to the loop-space question, more closely related to Stasheff's theory, but still expressible in terms of homotopy operations taking values in homotopy groups (rather than higher homotopies), will be described in a future paper, with a view to such an extension.

\section{References}

[A] J. F. Adams, The sphere, considered as an $H$-space mod $p$, Quart. J. Math. Oxford Ser. (2) 12 (1961), 52-60.

[B] H. J. Baues, Geometry of loop spaces and the cobar construction, Mem. Amer. Math. Soc. 230 (1980).

[Bl1] D. Blanc, A Hurewicz spectral sequence for homology, Trans. Amer. Math. Soc. 318 (1990), 335-354.

[B12] -, Abelian П-algebras and their projective dimension, in: M. C. Tangora (ed.), Algebraic Topology: Oaxtepec 1991, Contemp. Math. 146, Amer. Math. Soc., Providence, R.I., 1993, 39-48. 
[Bl3] D. Blanc, Higher homotopy operations and the realizability of homotopy groups, Proc. London Math. Soc. (3) 70 (1995), 214-240.

[Bl4] - Homotopy operations and the obstructions to being an H-space, Manuscripta Math. 88 (1995), 497-515.

[Bl5] -, Homotopy operations and rational homotopy type, preprint, 1996.

[BV] J. M. Boardman and R. M. Vog t, Homotopy Invariant Algebraic Structures on Topological Spaces, Lecture Notes in Math. 347, Springer, Berlin, 1973.

[BF] A. K. Bousfield and E. M. Friedlander, Homotopy theory of $\Gamma$-spaces, spectra, and bisimplicial sets, in: M. G. Barratt and M. E. Mahowald (eds.), Geometric Applications of Homotopy Theory, II, Lecture Notes in Math. 658, Springer, Berlin, 1978, 80-130.

[BK] A. K. Bousfield and D. M. Kan, Homotopy Limits, Completions, and Localizations, Lecture Notes in Math. 304, Springer, Berlin, 1972.

[BL] R. Brown and J.-L. Loday, Van Kampen theorems for diagrams of spaces, Topology 26 (1987), 311-335.

[C] E. B. Curtis, Simplicial homotopy theory, Adv. in Math. 6 (1971), 107-209.

[DL] A. Dold and R. K. Lashof, Principal quasifibrations and fibre homotopy equivalence of bundles, Illinois J. Math. 3 (1959), 285-305.

[DHK] W. G. Dwyer, P. S. Hirschhorn and D. M. Kan, Model categories and more general abstract homotopy theory, preprint, 1996.

[DKS] W. G. Dwyer, D. M. Kan and J. H. Smith, Homotopy commutative diagrams and their realizations, J. Pure Appl. Algebra 57 (1989), 5-24.

[F] M. Fuchs, A modified Dold-Lashof construction that does classify H-principal fibrations, Math. Ann. (2) 192 (1971), 328-340.

[G] R. Godement, Topologie algébrique et théorie des faisceaux, Act. Sci. \& Ind. 1252, Publ. Inst. Math. Univ. Strasbourg XIII, Hermann, Paris, 1964.

[H] P. J. Hilton, A remark on loop spaces, Proc. Amer. Math. Soc. 15 (1964), 596600.

[Hi] P. S. Hirschhorn, Localization of model categories, preprint, 1996.

[J] I. M. James, Reduced product spaces, Ann. of Math. (2) 62 (1955), 170-197.

[K] D. M. Kan, On homotopy theory and c.s.s. groups, ibid. 68 (1958), 38-53.

[Ka] R. M. Kane, The Homology of Hopf Spaces, North-Holland Math. Library 40, North-Holland, Amsterdam, 1988.

[M] S. MacLane, Categories for the Working Mathematician, Grad. Texts in Math. 5, Springer, Berlin, 1971.

[Ma1] J. P. May, Simplicial Objects in Algebraic Topology, Univ. Chicago Press, Chicago, 1967.

[Ma2] - The Geometry of Iterated Loop Spaces, Lecture Notes in Math. 271, Springer, Berlin, 1972.

[Mi1] J. W. Milnor, Construction of universal bundles, I, Ann. of Math. (2) 3 (1956), $272-284$.

[Mi2] —, On the construction FK, in: J. F. Adams (ed.), Algebraic Topology-A Student's Guide, London Math. Soc. Lecture Note Ser. 4, Cambridge Univ. Press, Cambridge, 1972, 119-136.

[N] J. A. Neisendorfer, Properties of certain H-spaces, Quart. J. Math. Oxford Ser. (2) 34 (1983), 201-209.

[Q1] D. G. Quillen, Homotopical Algebra, Lecture Notes in Math. 20, Springer, Berlin, 1963.

[Q2] - Spectral sequences of a double semi-simplicial group, Topology 5 (1966), $155-156$. 
[RS] C. P. Rourke and B. J. Sanderson, $\Delta$-sets I: Homotopy theory, Quart. J. Math. Oxford Ser. (2) 22 (1972), 321-338.

[S1] G. B. Segal, Classifying spaces and spectral sequences, Publ. Math. Inst. Hautes Etudes Sci. 34 (1968), 105-112.

[S2] - Categories and cohomology theories, Topology 13 (1974), 293-312.

[St1] J. D. Stasheff, Homotopy associativity of $H$-spaces, I, Trans. Amer. Math. Soc. 108 (1963), 275-292.

[St2] -, Homotopy associativity of $H$-spaces, II, ibid., 293-312.

[St3] - , H-spaces from a Homotopy Point of View, Lecture Notes in Math. 161, Springer, Berlin, 1970.

[St4] - $H$-spaces and classifying spaces: foundations and recent developments, in: A. Liulevicius (ed.), Algebraic Topology, Proc. Sympos. Pure Math. 22, Amer. Math. Soc., Providence, 1971, 247-272.

[Ste] N. E. Steenrod, Milgram's classifying space of a topological group, Topology 7 (1968), 349-368.

[Stv] C. R. Stover, A Van Kampen spectral sequence for higher homotopy groups, Topology 29 (1990), 9-26.

[Su] M. Sugawara, A condition that a space is group-like, Math. J. Okayama Univ. 7 (1957), 123-149.

[W] G. W. Whitehead, Elements of Homotopy Theory, Grad. Texts in Math. 61, Springer, Berlin, 1971.

[Z] A. Zabrodsky, Homotopy associativity and finite CW complexes, Topology 9 (1970), 121-128.

[Zi] G. M. Ziegler, Lectures on Polytopes, Grad. Texts in Math. 152, Springer, Berlin, 1995.

Department of Mathematics and Computer Science

University of Haifa

31905 Haifa, Israel

E-mail: blanc@mathcs2.haifa.ac.il

Received 16 September 1996;

in revised form 7 February 1997 